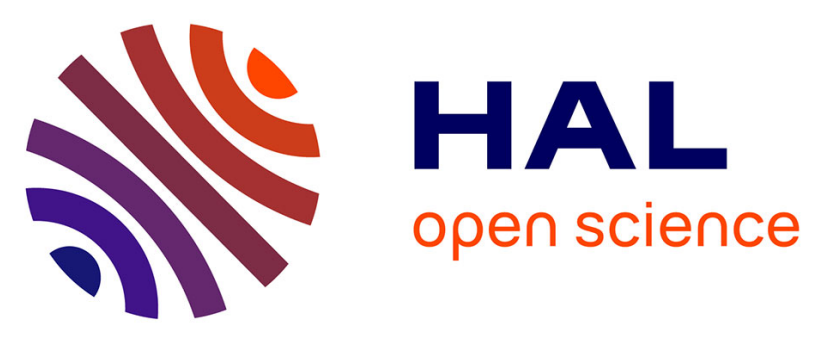

\title{
Les armes en fer d'Uxellodunum (Puy d'Issolud, Lot), dernière bataille de César en Gaule: étude paléométallurgique de pointes de flèche et trait de catapulte
}

\author{
Guillaume Renoux, Francis Dabosi, Jean-Marie Pailler
}

\section{To cite this version:}

Guillaume Renoux, Francis Dabosi, Jean-Marie Pailler. Les armes en fer d'Uxellodunum (Puy d'Issolud, Lot), dernière bataille de César en Gaule: étude paléométallurgique de pointes de flèche et trait de catapulte. Archeosciences, revue d'Archéométrie, 2004, 28 (1), pp.141-152. 10.3406/arsci.2004.1069 . hal-01952322

\section{HAL Id: hal-01952322 \\ https://hal.science/hal-01952322}

Submitted on 12 Dec 2018

HAL is a multi-disciplinary open access archive for the deposit and dissemination of scientific research documents, whether they are published or not. The documents may come from teaching and research institutions in France or abroad, or from public or private research centers.
L'archive ouverte pluridisciplinaire $\mathbf{H A L}$, est destinée au dépôt et à la diffusion de documents scientifiques de niveau recherche, publiés ou non, émanant des établissements d'enseignement et de recherche français ou étrangers, des laboratoires publics ou privés. 
Guillaume Renoux

Francis Dabosi

Jean-Marie Pailler

\section{Les armes en fer d'Uxellodunum (Puy d'Issolud, Lot), dernière}

bataille de César en Gaule : étude paléométallurgique de pointes de flèche et trait de catapulte

In: Revue d'Archéométrie, N²8, 2004. pp. 141-152.

Citer ce document / Cite this document :

Renoux Guillaume, Dabosi Francis, Pailler Jean-Marie. Les armes en fer d'Uxellodunum (Puy d'Issolud, Lot), dernière bataille de César en Gaule : étude paléométallurgique de pointes de flèche et trait de catapulte. In: Revue d'Archéométrie, N²8, 2004. pp. 141-152.

doi : 10.3406/arsci.2004.1069

http://www.persee.fr/web/revues/home/prescript/article/arsci_0399-1237_2004_num_28_1_1069 


\section{Abstract}

Excavations on the site of Uxellodunum have delivered an exceptional collection of iron weapons, with arrow heads and arrows of catapult. The major aim of this study concerns the characterization of their metallurgical structures and the determination of distribution, morphology and composition of phases and constitants. From that time, it is possible to suggest various ways for production and thermomechanical treatments developped by blacksmiths, at the 1st century BC ; This contributes to feed the technical history of forging.

Several steps are retained for observation and analysis ; $X$ rays radiography and metallographic analysis (macroscopy and microscopy -optical or scanning electronic MEB), were carried out for identifying the structural features of each type of component (metal, oxides, carbides or residual slag inclusions). Vickers microhardness values let to evaluate the level of mechanical resistance. Electron micro- probe and secondary ion mass spectrometrie (SIMS) were performed in order to reach local distribution of impurities at surface or, occasinaly inside (concentration profile), by ionic abrasionanalysis sequences.

Correlations between these results let to discriminate some specific data, able to bring a better identification of steps of smelting and forging processes involved in the operating chain, from the raw materials to these artefacts.

\section{Résumé}

Les fouilles effectuées sur le site d'Uxellodunum ont permis de révéler un mobilier exceptionnel d'armes en fer se composant de pointes de flèche et de fers de trait de catapulte. L'objectif principal de l'étude est de caractériser la microstructure, la distribution, la morphologie et la composition des phases présentes dans ces objets. Il est dès lors possible de proposer des protocoles d'élaboration et de transformation mis en oeuvre par les forgerons, au 1er siècle av. J.-C. pour la fabrication de ces armes. On contribue par là-même à nourrir l'histoire des techniques de forgeage.

Plusieurs étapes d'observation et d'analyse ont été retenues dont : la radiographie par RX des objets, l'examen métallographiquc macro- et microscopique -optique et électronique (MEB)-, permettant l'identification des structures du métal et des inclusions ou phases secondaires (oxydes, carbures, scories résiduelles). Des essais de microdureté Vickers ont permis d'apprécier le niveau de résistance mécanique des armes. Ces observations ont été complétées par des études microanalytiques à la microsonde électronique et par spectrométrie d'émission d'ions secondaires (SIMS) ; on atteint alors la distribution locale des divers éléments, soit, en surface, soit en profondeur lors de séquences d'abrasion-analyse.

La corrélation des résultats obtenus doit conduire, à terme, à dégager un certain nombre d'indices discriminatoires et permettre d'expliciter les données relatives aux différentes étapes de la chaîne opératoire, de l'élaboration du métal à la réalisation des armes. 


\title{
Les armes en fer d'Uxellodunum (Puy d'Issolud, Lot), dernière bataille de César en Gaule : Etude paléométallurgique de pointes de flèche et trait de catapulte
}

\author{
Guillaume RENOUX*, Francis DABOSI** et Jean-Marie PAILLER*
}

\begin{abstract}
Résumé : Les fouilles effectuées sur le site d'Uxcllodunum ont permis de révéler un mobilier exceptionnel d'armes en fer se composant de pointes de flèche et de fers de trait de catapulte. L'objectif principal de l'étude est de caractériser la microstructure, la distribution, la morphologie et la composition des phases présentes dans ces objets. Il est dès lors possible de proposer des protocoles d'élaboration ct de transformation mis en oeuvre par les forgerons, au Ier siècle av. J.-C. pour la fabrication de ces armes. On contribue par là-même à nourrir l'histoire des techniques de forgeage.

Plusicurs étapes d'observation et d'analyse ont été retenues dont : la radiographie par RX des objets, l'examen métallographique macroet microscopique -optique et électronique (MEB)-, permettant l'identification des structures du métal et des inclusions ou phases secondaires (oxydes, carbures, scories résiduelles). Des essais de microdureté Vickers ont permis d'apprécier le niveau de résistance mécanique des armes. Ces observations ont été complétées par des études microanalytiques à la microsonde électronique et par spectrométrie d'émission d'ions secondaires (SIMS); on atteint alors la distribution locale des divers ćléments, soit, en surface, soit en profondeur lors de séquences d'abrasion-analyse.

La corrélation des résultats obtenus doit conduirc, à terme, à dégager un certain nombre d'indices discriminatoires et permettre d'expliciter les données relatives aux différentes étapes de la chaîne opératoire, de l'élaboration du métal à la réalisation des armes.

Abstract : Excavations on the site of Uxellodunum have delivered an exceptional collection of iron weapons, with arrow heads and arrows of catapult. The major aim of this study concerns the characterization of their metallurgical structures and the determination of distribution, morphology and composition of phases and constitants. From that time, it is possible to suggest various ways for production and thermo-mechanical treatments developped by blacksmiths, at the 1st century BC; This contributes to feed the technical history of forging.

Several steps are retained for observation and analysis; $X$ rays radiography and metallographic analysis (macroscopy and microscopy -optical or scanning electronic MEB), were carricd out for identifying the structural features of each type of component (metal, oxides, carbides or residual slag inclusions). Vickers microhardness values let to evaluatc the level of mechanical resistance. Electron microprobe and secondary ion mass spectrométrie (SIMS) were performed in order to reach local distribution of impurities at surfacc or, occasinaly inside (concentration profile), by ionic abrasion-analysis sequences.

Correlations between these results let to discriminate some specific data, able to bring a better identification of steps of smelting and forging processes involved in the opcrating chain, from the raw materials to these artefacts.
\end{abstract}

Mots-clés : Uxellodunum, armement, pointes de flèches, trait de catapulte, forgerons, histoire des techniques, métallographie, microanalyses.

Key-words : Uxellodunum, iron weapons, arrows heads, arrow catapult, technical history, blacksmiths, mctallography, microanalysis.

La métallurgie remarquablement maîtrisée par les sociétés anciennes constitue un excellent champ de recherche susceptible de favoriser leur connaissance. L'histoire de l'humanité ne peut être que le reflet d'un long développement de l'esprit, des métiers et des techniques. H. J. Hundt dans sa Correspondance écrivait qu' « en comprenant les progrès dans les métiers, nous comprenons un peu de l'esprit de l'artisan et bien plus de l'esprit d'une culture antique ». Quels objets fabriqués pouvaient, dans ce cas, être plus représentatifs des avancées de la métal- lurgie qu'un mobilier d'armes dont la nature et le nombre devaient être sans cesse renouvelés et les performances améliorées? Les différentes guerres que se sont livrées les hommes, à travers l'histoire, ont contribué à favoriser l'amélioration des techniques et notamment celles de la forge. Dans ces conditions, les découvertes faites au Puy d'Issolud (Lot) nous offrent un terrain favorable à l'étude de ces techniques. En effet, les fouilles effectuées sur ce site, depuis sa première exploration en 1850 jusqu'à celles poursuivies de nos jours par J.-P. Girault, ont

\footnotetext{
*U.M.R. 5608, C.N.R.S, Unité Toulousaine d'Archéologie et d'Histoire, Université de Toulouse -Le Mirail. guillaume.renoux@free.fr et paillcr.jcanmarie@wanadoo.fr

** U.M.R. 5085, C.N.R.S., C.I.R.I.M.A.T., E.N.S.I.A.C.E.T., Institut National Polytechnique de Toulouse. dabosi.francis@wanadoo.fr
} 
permis de révéler un exceptionnel mobilier d'armes en fer se composant notamment de pointes de flèche et de traits de catapulte ${ }^{1}$. Notre étude, engagée sur une partie du matériel, aborde l'examen métallurgique de ces types d'objets.

L'objectif principal est de caractériser, pour ces armes, les structures métallurgiques, les distributions, les morphologies et compositions des phases et des constituants présents. Il est, dès lors, possible d'imaginer les différents protocoles d'élaboration et les traitements thermo-mécaniques développés par les forgerons au $\mathrm{I}^{\mathrm{cr}} \mathrm{s}$. av. J.-C. pour leur mise en forme, et d'apporter de nouveaux éléments à une histoire des techniques de la forge.

Ce travail faisant appel à différentes méthodes d'étude métallographique conduit à poser un certain nombre de questions. La connaissance intime de ces objets peut-elle nous permettre d'identifier leur mode de fabrication et d'expliciter leur provenance ? Peut-on identifier la nature du minerai de fer utilisé ? Maîtrisait-on les techniques de forgeage et les traitements (cémentation, trempe)?

\section{Le Puy d'Issolud : Contexte géographique et historique, exploration archéologique}

Le Puy d'Issolud se situe dans le département du Lot entre le Haut-Quercy, le Périgord Noir et les Causses de Gramat et de Martel (fig. 1). Dominant les vallées de la Dordogne, de la Tourmente et de la Sourdoire, il forme une butte-témoin, dont le sommet atteint $311 \mathrm{~m}$, au lieu dit Los Temples. A ses pieds naît une source, nommée Fontaine de Loulié.

Depuis le milieu du $\mathrm{XIX}^{\mathrm{c}}$ siècle, les vestiges mis au jour au Puy d'Issolud ont contribué à identifier ce site comme étant l'antique oppidum d'Uxellodunum ${ }^{2}$. En effet, le Puy d'Issolud présente par rapport aux écrits antiques de nombreuses similitudes avec le lieu du dernier combat entre les Romains et les Gaulois ${ }^{3}$.

Nous retracerons brièvement les phases principales de ce conflit relatées dans le Livre VIII de la Guerre des Gaules de César, livre écrit par Hirtius, un de ses lieutenants ${ }^{4}$. Certains aspects de notre démarche scientifique ne peuvent, en effet, ignorer le contexte spécifique des événements et de leurs conséquences prévisibles sur l'altération des microstructures et propriétés des armes enfouies à l'issue des combats.

La défaite gauloise d'Alésia en 52 av. J.-C. plonge la Gaule tout entière dans un profond désarroi, jusqu'au jour, où, en 51 av. J.-C., un ancien combattant d'Alésia, Drappès, aidé par le Cadurque Luctérios, parvient à regrouper une armée de 2 à 5000 hommes pour s'em- parer de la Provincia. Ils sont, cependant, rejoints assez rapidement par deux légions romaines leur barrant le chemin. Menacés par l'approche de ces armées, Drappès et Luctérios décident de se réfugier sur l'oppidum d'Uxellodunum sur le territoire des Cadurques. Au cours d'une opération de ravitaillement, Drappès est capturé et Luctérios doit fuir. Cette nouvelle défaite n'entame pas pour aulant la détermination des Gaulois de l'oppidum, qui se trouve bientôt entouré de fortifications construites par le «Génie» romain.

César, venu en personne sur le site, constate très vite que la seule solution pour se rendre maître de la place forte consiste à priver d'eau potable ses occupants. Dans ce but, il établit à des endroits stratégiques des archers, des frondeurs et des machines de guerre pour interdire aux assiégés l'accès d'une rivière qui coule au pied de la montagne escarpée, où ils vont puiser l'eau nécessaire pour leur survie.

Les Gaulois, s'apercevant du stratagème, décident alors de s'approvisionner au « seul endroit encore accessible, au pied même du mur de la ville, où jaillit une source abondante du côté que laisse libre le circuit de la rivière $»^{5}$.

Désormais le seul moyen dont dispose César pour conquérir l'oppidum est de détourner la source de son lit. Il entreprend donc de faire creuser « des conduits souterrains dans la direction où les filets d'eau et la source aboutissent $»^{6}$. En même temps, César ordonne la construction d'un terrassement destiné à supporter le poids d'une tour

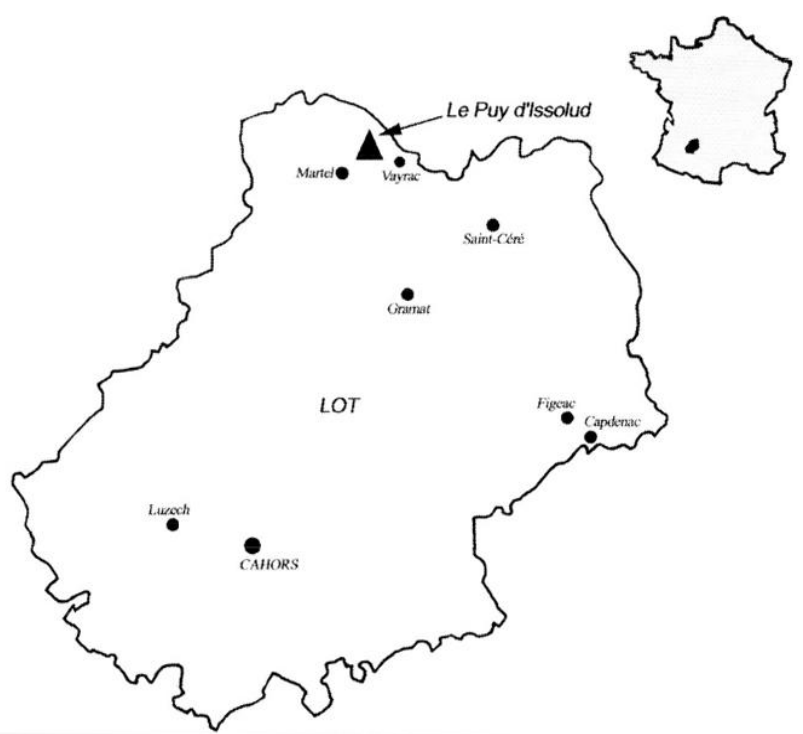

Figure 1 : Situation gćographique du Puy d'Issolud (Lot). Figure I: Geographic position of the site of The Puy d'Issolud (Lot).

\footnotetext{
' Girault, J.-P., Billiant, P., Puy d'Issolud, histoire des recherches et découvertes archéologiques (cn cours de publication dans Aquitania).

${ }^{2}$ Lors des Journées César, cn avril 2001, organisćcs à l'Universitć de Toulousc-Le Mirail, Christian Goudineau, professcur au Collège de France, a reconnu après bien d'autres le Puy d'Issolud comme étant Uxellodunum.

${ }^{3}$ Trois autres sites, se situant dans le Lot, se disputent depuis la parution de l'ouvrage de Camille Jullian, Histoire de la Gaule, en 1909, l'identification d'Uxellodunum à Luzech, Capdenac et Murcens.

${ }^{4}$ César, Commentarii de Bello Gallico, Livre VIII, 21-44, texte établi ct traduit par L.A. Constant, Paris, Les Belles Lettres, C.U.F., 1996. Ce dernicr livre de la Guerre des Gaules fut rédigé par un des officiers de César, Aulus Hirtius. Il fut consul cn même temps que Vibius Pansa en 43 av. J.-C. Tous deux prirent les armes contre Marc-Antoine et périrent lors du siège de Modène (Mutina). Certains pensent qu'il a probablement aussi écrit le Bellum Alexandrinum, suite de la Guerre Civile de César (48-47 av. J.-C.).

${ }^{5} B . G .$, VIII, 41 .

${ }^{6}$ B.G., VIII, 41.
} 
permettant de dominer la source. Du haut de celle-ci, les Romains harcèlent les Gaulois grâce à « des machines qui lancent des projectiles sur les abords de la fontaine $»^{7}$. Mais la riposte des assiégés ne se fait pas attendre. Les Gaulois envoient sur les positions romaines des tonneaux enflammés remplis de suif, de poix et de minces lattes de bois. Un incendie d'une très forte ampleur fait rage du côté des Romains ; les cohortes de César parviennent cependant à maîtriser le feu, sans que, pour autant, l'opiniâtreté et la résistance gauloise s'en trouvent diminuées. Le siège d'Uxellodunum prend fin quand les « ruisselets qui alimentent la source sont coupés par les canaux souterrains et détournés de leur cours $»^{8}$. Les Gaulois, inévitablement condamnés à mourir de soif et croyant à une intervention divine, se livrent au proconsul.

Ainsi s'achève le siège d'Uxellodunum, l'un des derniers épisodes majeurs de la Guerre des Gaules que l'oubli n'a pu effacer grâce aux recherches archéologiques engagées dès 1850 et poursuivies jusqu'à nos jours en divers lieux et enfin avec succès au Puy d'Issolud.

Les toutes premières études sur le terrain sont entreprises, en 1865, par J.-B. Cessac à la Fontaine dite de Loulié au pied du Puy d'Issolud; Cessac pense, en effet, que cette fontaine correspond bien aux indications fournies par le récit antique. Sa persévérance et ses efforts sont très vite récompensés puisque, entre mai et juin 1865 , il peut mettre au jour les traces d'un incendie et surtout découvrir une galerie artificielle sur une profondeur de 40 mètres, comportant encore ses étais. Dans le même temps des traits de catapulte et des pointes de flèche sont recueillis, ainsi que des galets ayant servi de balles de fronde.

Napoléon III est convaincu de la validité de ces découvertes qui, déclare-t-il dans son Histoire de Jules César, ne laissent plus aucun doute sur l'emplacement d'Uxellodunum au Puy d'Issolud'. Les campagnes de fouilles se succèdent. Il faut cependant attendre 1990 pour que J.P. Girault entreprenne, sous la responsabilité du Service Régional de l'Archéologie de Midi-Pyrénées, de nouvelles investigations particulièrement fructueuses, grâce à l'emploi de méthodes de fouille rigoureuses. Les anciens fouilleurs qui se sont succédé à la fontaine de Loulié ont détruit une partie du site, en faisant des tranchées très profondes. Il subsiste cependant des buttes témoins, qui nous révèlent des aménagements et des structures archéologiques en place. Les fouilles de la butte BU10 ont permis de mettre en évidence une couche de destruction provenant d'un aménagement gaulois incendié par les Romains. La présence d'une assez importante zone de terre rubéfiée (calcinée par endroits), de bûches carbonisées, de briques cuites lors de l'incendie (crues à l'origine), des fragments d'amphores (Dressel 1b), de la céramique indigène gauloise, des clous de galicae et d'armement romain ont permis de parfaitement dater cette structure ${ }^{10}$. Les recherches sur la butte BU1 ont mis au jour un sol gaulois interrompu contre de gros blocs côté sud (il pourrait s'agir d'un reste de fortification). Sur ce sol, il a été mis au jour des pointes de flèche, des fers de traits de catapulte ainsi que des fragments d'amphores.

Ce travail de terrain, toujours en cours, s'accompagne d'une étude sur le passé du Puy d'Issolud, répertoriant aussi toutes les trouvailles archéologiques anciennes et récentes. L'armement arrive au premier plan de toutes ces découvertes. Il est d'une remarquable richesse, tant par sa quantité que par son état de conservation, et d'un exceptionnel intérêt par sa datation très précise au milieu $\mathrm{du} \mathrm{I}^{\mathrm{er}} \mathrm{s}$. av. J.-C. C'est ce mobilier que nous nous sommes proposé d'étudier, pour en définir l'identité et les propriétés et tenter de reconstituer les conditions techniques de sa fabrication.

\section{L'échantillonnage étudié}

Le mobilier archéologique en fer retrouvé au Puy d'Issolud est, nous l'avons dit, de par son importance, unique. Nous avons été contraints de ce fait d'opérer, pour nos études métallographiques, une sélection représentative de l'ensemble des pointes de flèches et des fers de trait de catapulte. Notre choix s'est établi en fonction de trois critères :

- la typologie des pointes de flèche : droites ou à barbelure.

- leur position stratigraphique lors des fouilles.

- leur étal de conservation.

J.-P. Girault a pu recenser environ 900 pointes de flèche, découvertes depuis 1850 . Sur ce total, 620 proviennent de la fontaine de Loulié ; 210 d'entre elles sont cependant en mauvais état ou fragmentées et ne peuvent faire l'objet d'une identification fiable et sûre. Enfin, 13 pointes de flèche ont été découvertes à ce jour sur le plateau du Puy d'Issolud.

Si l'on se réfère à la typologie des pointes de flèche mise au point par Alain Duval (Duval, 1970), ces différents spécimens se répartissent ainsi :

- 260 flèches droites,

- 137 flèches à une barbelure ,

- 3 flèches à un aileron,

- 4 flèches à deux ailerons,

- 5 flèches, enfin, à fer foliacé avec deux bords tranchants et sans aileron. Tous ces types sont à douille.

Les fers de trait de catapulte, armes typiquement romaines, sont, quant à eux, beaucoup plus rares dans les découvertes faites en fouille. Sur le site de la Fontaine de Loulié, 70 fers de traits à douille de catapulte ont été découverts ${ }^{11}$. Ils présentent une pointe pyramidale et une

\footnotetext{
${ }^{7}$ B.G., VIII, 42.

${ }^{8}$ B.G., VIII, 43.

${ }^{9}$ Napolćon III, Histoire de Jules César, Paris, 1866, II, p. 343-347.

${ }^{10}$ Des fourchettes de datation ont pu être proposées à partir de bûches carbonisées retrouvées sur le sol rubéfić. Les résultats nous donnent un intervalle allant de 103 av. J.-C. à 67 ap. J.-C. Deux datations par archéomagnétisme (Institut de Physique du globe de Paris) provenant du sol rubéfié donnent des intervalles d'âge possible allant de 100 av. J.-C. à 10 ap. J.-C. et de 100 à 5 av. J.-C. Ces fourchettes sont presque idéalement compatibles avec les conclusions chronologiques dictées par l'identification du lieu de la bataille (51 av. J.-C.).

"Notons qu'au temps de César, le terme de catapulte (catapulta) désigne la machine qui lançait des flèches et celui de baliste (balista), la machine lançant des pierres; cf. Vitruve, De Architectura, Les Belles-Lettres, Paris, 1986, Livre X, 10-12. Plusicurs sic̀cles plus tard le mot catapulte disparaît pour ĉtre remplacé par baliste. Il y a cu interchangeabilité des deux termes.
} 
base quadrangulaire dégagée d'une courte tige cylindrique ouverte à la base. Nos études métallographiques portent sur des spécimens de chaque type, le tableau 1 récapitule les échantillons, mis à notre disposition par J.P. Girault, qui ont fait déjà l'objet d'analyses ; le reste est en cours d'étude.

\section{Méthodes d'étude expérimentales}

Tout mobilier ferreux ancien pose plusieurs problèmes de caractérisation. Enfouis depuis des siècles dans le sol, les objets archéologiques en fer sont recouverts d'une couche d'oxydes complexe plus ou moins importante, masquant (voire détruisant) leur structure et leur type originels. La seule observation de la surface naturelle de l'objet exhumé présente, de ce fait, un intérêt limité vis-à-vis de la recherche de données à caractère technologique et scientifique sur les modes d'obtention ou de transformation thermomécanique (forgeage, par exemple) du matériau métallique qui constitue cet objet. Cela ne signifie évidemment pas que la nature de ces couches, leur importance et leur distribution, soient à ignorer ; leur connaissance est indispensable pour définir la surface originelle de l'objet ; par leur spécificité, elles peuvent, en outre, permettre de révéler certains «accidents de parcours» liés à des conditions particulières d'environnement (surchauffes associées à des incendies, par exemple) ou à des hétérogénéités structurales (zones soudées, corroyage irrégulier), comme nous le constaterons par la suite.

- La radiographie $\boldsymbol{X}$ de ces objets doit précéder tout autre examen ou analyse scientifique. Cette première étape nous a apporté des informations précieuses sur la morphologie interne des produits étudiés. Si elle permet de déceler la présence de décors, de soudures..., elle sert surtout à mieux différencier les zones métalliques résiduelles des couches d'oxydes ou d'altérations diverses; de ce fait, elle permet de prélever avec beaucoup plus de sécurité et d'efficacité les échantillons à étudier. Mais dans le cas présent, la radiographie $\mathrm{X}$ n'a cependant pas été véritablement déterminante dans l'étude des pointes de flèche et traits de catapulte sélectionnés. En effet, grâce au nombre considérable de ces armes retrouvées au Puy d'Issolud, il nous était loisible d'examiner tout le volume des objets retenus sans avoir à nous limiter à des prélèvements très sélectifs et peu nombreux (Pajot, 1978 ; Mohen, 1973). Il était donc possible, en général, de passer rapidement à l'étude structurale.

- L'examen structural des échantillons a nécessité une préparation métallographique classique :

Le découpage mécanique soigné à la scie diamantée a permis de sélectionner les zones privilégiées d'observation, à savoir la tête et la douille (chaque échantillon découpé offre une surface d'observation généralement comprise entre 0,1 et $1 \mathrm{~cm}^{2}$, qu'il s'agisse de coupes transversales ou longitudinales). Cette première étape en apparence triviale nécessite en fait beaucoup de soins. Certaines précautions doivent, en effet, être prises pour éviter toutes modifications intempestives de la structure interne du métal qui pourraient être provoquées, soit par un échauffement, soit par une déformation du métal entraînant un écrouissage. L'échantillon est alors enrobé dans une résine polymérisable à froid puis poli mécaniquement : prépolissage aux papiers abrasifs (grades 80

\begin{tabular}{|c|l|l|}
\hline $\begin{array}{c}\text { Description de l'objet } \\
\text { Pointe de flèche à une barbelure }\end{array}$ & Numéro & \multicolumn{1}{|c|}{ Provenance } \\
\hline Pointe de flèche à une barbelure & BU10-438 & $\begin{array}{l}\text { Fouille 1999. Butte BU10, sol gaulois. Zone } \\
\text { incendiée avec importantes traces de } \\
\text { rubéfaction. }\end{array}$ \\
\hline Pointe de flèche à une barbelure ou droite & BU10-706 & $\begin{array}{l}\text { Fouille 1999. Butte BU10, sol gaulois. Zone } \\
\text { incendiée avec importantes traces de } \\
\text { rubéfaction. }\end{array}$ \\
\hline Fouille 1999. Butte BU10, sol gaulois. Zone \\
incendiée avec importantes traces de \\
rubéfaction.
\end{tabular}

Tableau 1 : Récapitulatif des pointes de flèche et du fer de trait de catapulte du Puy d'Issolud ayant fait l'objet d'une étude métallographique. 
à 4000) puis polissage de finition à la pâte diamantée (6 $\mu \mathrm{m}$ sur drap dur et $1 \mu \mathrm{m}$ sur drap feutré). Au terme de ce travail, déterminant pour la suite, l'on obtient un «poli miroir», sur lequel il est déjà possible d'effectuer un examen préalable. Les fissures, cavités et inclusions sont clairement détectées. Cependant la microstructure ne sera accessible que par attaque chimique, à l'aide d'un réactif chimique spécifique. Nous avons retenu le nital $2 \%$ ( $2 \%$ d'acide nitrique dans l'alcool éthylique) qui est employé de façon classique pour révéler la microstructure des aciers au carbone ou faiblement alliés. Le protocole de préparation achevé, les examens métallographiques peuvent être effectués (Serdon, 2002 et 2003).

- Une première observation macroscopique à la loupe binoculaire révèle, à faible grossissement, le fibrage éventuel de l'échantillon et la distribution des inclusions (des particules de scories pour l'essentiel) dans la matrice métallique.

- L'étude en microscopie optique intervient à une échelle beaucoup plus fine (grossissement de 100 à 1200) et révèle la microstructure de l'objet : nature, morphologie et distribution des phases, tant au niveau de la matrice métallique qu'à ceux des scories et des couches de corrosion, préservées lors de la découpe.

- Pour atteindre enfin des grossissements beaucoup plus élevés, nous avons eu recours au microscope électronique à balayage (M.E.B.). L'intérêt de celui-ci réside dans son pouvoir très important de résolution et sa grande profondeur de champ; des analyses ponctuelles sur des surfaces de l'ordre du $\mu \mathrm{m}^{2}$ sont rendues possibles par l'analyse dispersive des rayons $\mathrm{X}$ en énergie (méthode EDXS). Les spectres de pics obtenus sont caractéristiques des éléments (de numéro atomique supérieur à 10) présents dans le matériau ; une estimation quantitative de leurs teneurs nécessiterait l'usage d'étalons.

- La microsonde ionique (spectroscopie d'émission d'ions secondaires), grâce à l'interaction d'ions (argon, césium) avec la surface de l'échantillon, donne naissance à des émissions secondaires : photons, électrons, atomes, ions...caractéristiques de tous les éléments présents en surface et de leur distribution (quel que soit leur numéro atomique), ces émissions confèrent à cette technique un grand intérêt analytique ; dans le cas des objets ferreux, elle est particulièrement adaptée à l'identification simultanée des métalloïdes $(\mathrm{C}, \mathrm{N}, \mathrm{O}, \mathrm{Cl}, \mathrm{S})$ présents dans le métal ou dans les inclusions. Même en mode analyse de surface (ions primaires d'assez faible énergie), le "pelage» incessant de la surface-cible de l'échantillon conduit à considérer qu'il s'agit d'un test destructif.

- La diffraction de rayons $X$, de son côté, s'est révélée très utile pour l'étude analytique des couches de corrosion, tandis que les mesures de microdureté (Vickers ou, éventuellement, Knoop sous une charge réduite de $50 \mathrm{~g}$ ) permettent de fixer le niveau relatif des propriétés mécaniques des diverses zones examinées de la pièce.

\section{Observations et résultats}

\subsection{Les radiographies}

Précédant - comme nous l'avons déjà souligné- les examens métallographiques, les radiographies ont permis de révéler la bonne, voire la très bonne, conservation de ces armes, favorisée à l'évidence par des conditions propices d'enfouissement naturel dans des argiles et des marnes, à l'abri d'une humidité trop importante. Il convient cependant de nuancer cette constatation. En effet, certaines pointes de flèche ont, comme le révèle le contexte de leur découverte dans un environnement argileux rubéfié lié à un épisode d'incendie intense, été soumises à des températures élevées et à une forte oxydation (cf. tab. 1); elles ne conservent qu'un faible volume de métal à cœur. Les zones oxydées y sont plus développées et la pointe peut même être totalement minéralisée. Les zones métalliques, en ce qui concerne les flèches, varient donc entre $0,1 \mathrm{~cm}$ et $0,5 \mathrm{~cm}$ d'épaisseur, $1,6 \mathrm{~cm}$ et 2,7 $\mathrm{cm}$ de longueur, $0,1 \mathrm{~cm}$ et $0,5 \mathrm{~cm}$ de large. Le trait de catapulte, quant à lui, renferme une plus grande masse de fer, longue de $2,6 \mathrm{~cm}$, large de $1,1 \mathrm{~cm}$ et épaisse de $1 \mathrm{~cm}$. Son état de conservation est excellent, avec un degré de corrosion très nettement inférieur à celui observé sur les pointes de flèche. Les conditions d'enfouissement et les techniques de forgeage peuvent très certainement expliquer cet état de conservation (fig. 2). Les zones les plus claires, observées sur ces images négatives au cœur des objets, correspondent au noyau métallique résiduel.

\subsection{Etudes métallographiques}

Nous pouvons, en préambule, dégager quelques caractéristiques générales. La microstructure se révèle très hétérogène, non seulement d'une flèche à l'autre mais aussi au sein d'un même objet, plus particulièrement en ce qui concerne les teneurs locales présumées en carbone. Enfin de nombreuses inclusions, notamment de scories plus ou moins organisées, sont observées au cœur du métal (Serdon, 2002).

$\mathrm{Au}$ delà de la simple description externe de l'objet (typologie), les examens métallographiques conduisent à la définition de structures typiques, allant d'un fer très doux, à très faible teneur en carbone, à des aciers plus ou moins riches en carbone ; la composition eutectoïde ( $0,8 \%$ poids de carbone) constitue, pour l'ensemble des objets examinés, une limite supérieure. La diversité des conditions thermiques et chimiques (carburation volontaire, décarburation accidentelle, etc...) rencontrées durant la mise en forme et la vie de ces objets se caractérise par des structures variées et typiques : ferritique, ferrito-perlitique, Widmanstätten, bainitique et martensitique. Ainsi recueille-t-on des indices significatifs sur les techniques probablement mises en œuvre pour la fabrication de ces pointes de flèches et trait de catapulte.

La structure ferritique, correspondant à un fer presque pur (moins de $0,02 \%$ en poids de $C$ ), possède une grande plasticité mais une assez mauvaise résistance mécanique et par conséquent se déforme facilement (fig. 3a) (Fluzin et al., 1983). Cette structure se rencontre sur la plupart des pointes de flèche. Dans la grande majorité des cas, elle se situe au cœur de celles-ci. Mais pour la flèche BU10-484 elle constitue, au contraire, l'essentiel de sa microstructure. Il s'agit ici, selon nous, d'un phénomène de décarburation en milieu oxydant à une température très élevée pouvant être liée à un incendie, sur lequel 

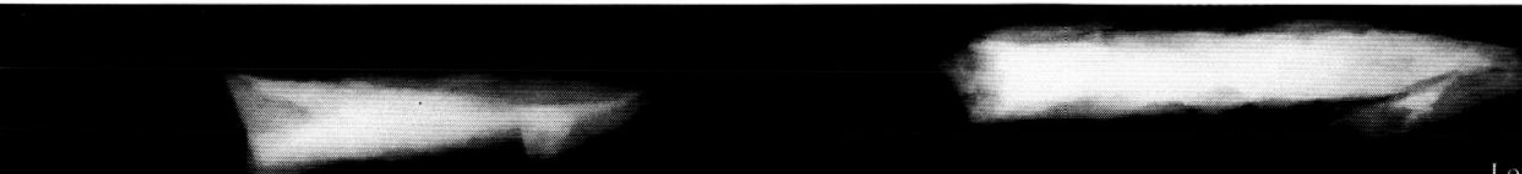

Loulié 2000

PM886

Loulié 2000

BU $1-n^{\circ} 20$

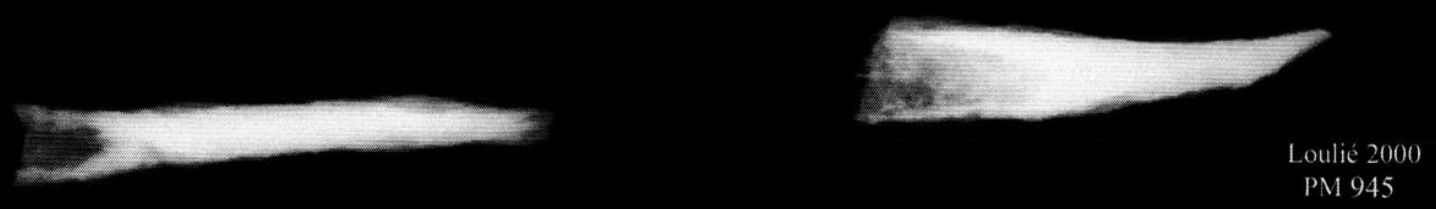

Loulie 2000

PM 1054

Sujet : radiographie (négatif) : vue de profil.

Clichés\&photo-interprétations : F. Séguy, C. Gargam - Atelier de restauration de la ville de Toulouse.

Figurc 2 : Excmples de radiographics des différents types de pointes de flèche. S'agissant de négatifs, les zones les plus claires des radiographies révèlent le métal résiduel plus absorbant que les oxydes.

Figure 2: X rays radiographs of the different types of arrow heads. With these negative photos, the clearest zones are associated to residual metal which is more absorbant than oxides.

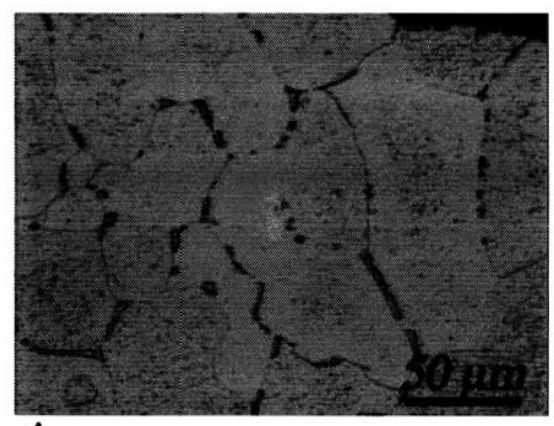

$A$

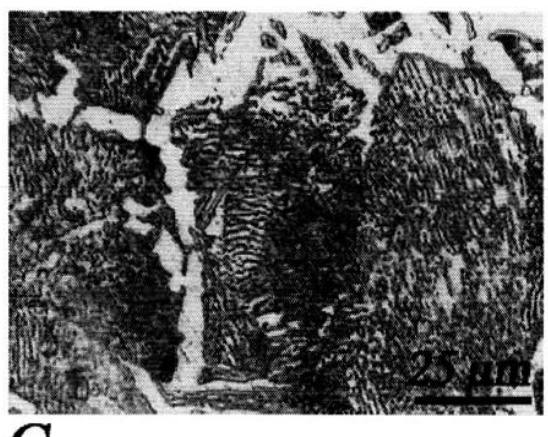

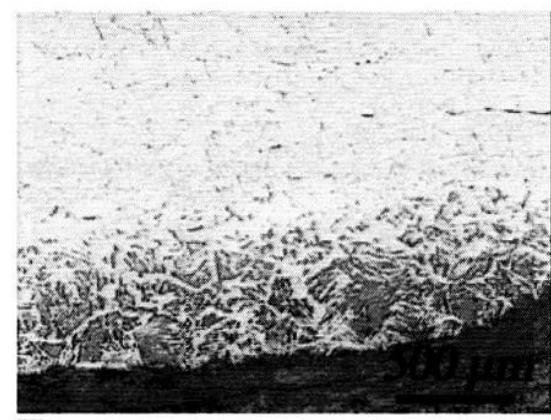

B

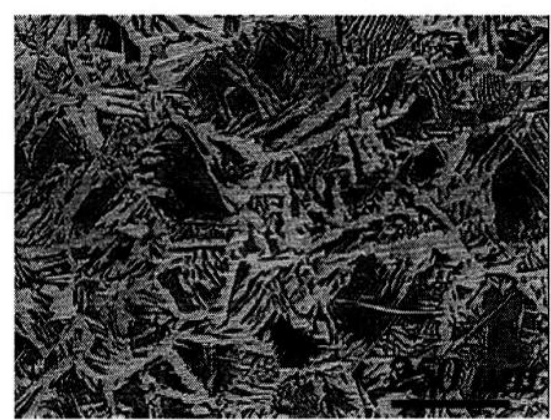

D

Figure 3 : Micrographies optiques. A : Pointe de flèch à une barbelure BU10-484. Corps de la flèche. Structure ferritique avec précipités de cémentitc aux joints de grains des cristaux de ferrite. B : Pointe de flèche à une barbelure PM 594. Bord de la barbelure. Structure ferrito-perlitique en surface ct structure ferritique à cour. C : Trait de catapulte. Corps du trait. Structure ferrito-perlitique ; cristaux primaires de ferrite (clairs) et structurc perlitique lamellaire (la cémentite constitue la phase sombre). D : Trait de catapulte. Exemple de structure de Widmanstätten.

Figure 3: Optical micrographs. A: Arrow head with one barbelure BU10-484. Frame of the arrow. Ferritic structure with precipitation of cementite at grain boundaries of ferrite crystals. B: Arrow head with one barbelure PM 594. Edge of the barbelure. Ferrito-perlitic structure in surface; ferritic structure in core. C: Arrow of catapult. Frame part. Ferrito-perlitic structure; primary crystals of ferrite (clear) and lamellar perlitic structure (cementite is the dark phase). D: Arrow of catapult. An example of Widmannstätten structure. 
nous devons nous attarder. Pour améliorer la résistance à la déformation, la dureté et, de ce fait, le pouvoir de pénétration de la pointe de flèche, il est certes possible de durcir le fer doux (très peu carburé) par un vigoureux forgeage à froid ; dans ce cas, on observe généralement des macles de déformation dans les cristaux de ferrite et des valeurs élevées de la dureté. Ce ne fut pas le cas pour cette pointe de fièche. Aucune macle ne fut observée et les valeurs de microdureté Vickers $\mathrm{Hv}$, sous une charge de $50 \mathrm{~g}$, demeurent faibles et comprises entre 81 et 143 . Nous considérons donc que cette pointe de flèche, probablement aciérée et performante quand elle fut utilisée, subit ensuite, durant l'incendie, une décarburation accidentelle totale générant une structure ferritique, équiaxe et recristallisée, de dureté médiocre. Des observations semblables ont été faites sur des épées celtiques (Uran, 1986 ; Lejars, 1989).

De plus cette structure ferritique comporte des précipités intergranulaires fins et globulaires de cémentite, caractéristiques d'un maintien prolongé dans un domaine de températures compris entre $400^{\circ} \mathrm{C}$ et $600^{\circ} \mathrm{C}$; localement des chapelets de particules sphéroïdales de cémentite ont coalescé sous forme de précipités plus gros et plus stables. Cette coalescence n'est rendue possible que si la pièce est exposée pendant une assez longue durée à une température élevée ; cela favorise les processus de diffusion atomique dans la matrice métallique, du carbone notamment. Ce faisceau de résultats est en accord avec les données des fouilles de 1999 à la Fontaine de Loulié (découverte de cette pointe de flèche BU10-484 dans un sol rubéfié) et avec les textes, mentionnant l'existence d'un long et rude incendie durant le siège d'Uxellodu$n_{u m^{12}}$. Les autres flèches découvertes sur cette zone ne comportent malheureusement plus de fer, ce qui interdit la comparaison.

L'analyse diffractométrique aux rayons $\mathrm{X}$ de prélèvements des zones oxydées de la flèche nous permet de renforcer cette hypothèse d'une décarburation accidentelle de cette arme. Parmi les phases décelées, figure la Wüstite. Cette dernière ne peut pas, thermodynamiquement, résulter de la seule exposition de cette flèche à l'environnement naturel ambiant (argile et marne) durant près de deux millénaires ; en revanche, une exposition prolongée à haute température (au-dessus de $570^{\circ} \mathrm{C}$, au moins) peut justifier son existence. En milieu oxydant chaud, l'incidence d'une décarburation de l'alliage et d'une oxydation postérieure à chaud devient plausible.

Les pointes de flèche PM 502, PM 662, PM 594, PM1054 et le trait de catapulte sont pour l'essentiel de structure ferrito-perlitique, (fig. $3 b$ et c) homogène dans les parties superficielles des flèches avec ou sans barbelures (ferrite primaire développée au niveau des exjoints de l'austénite ; la structure lamellaire classique de la perlite, avec l'alternance de lamelles de ferrite et de cémentite, est aisément observable au cœur des grains). Le cœur des pièces est en revanche ferritique, conférant ainsi aux flèches une certaine malléabilité. Il faut noter que la transition entre ces deux domaines se fait toujours progressivement. Cela conduit à confirmer le fait que la cémentation était pratiquée au ${ }^{\mathrm{cr}}$ siècle av. J.-C., afin d'améliorer la dureté superficielle des pointes de flèche et d'augmenter ainsi leur capacité de pénétration. Rappelons que le traitement de cémentation du fer par le carbone consiste à enrichir les zones superficielles de l'objet dans un environnement carburant. Il s'agit d'un traitement thermo-chimique de diffusion du carbone dans le réseau cristallin du fer à une température appropriée (souvent autour de $900^{\circ} \mathrm{C}$ ) pendant des durées d'autant plus longues que la profondeur de diffusion visée est élevée. Cela pouvait être réalisé, par exemple, par immersion de la pièce dans le charbon de bois en combustion libre dans un foyer de forge.

S'il est vrai que les conditions d'enfouissement ont favorisé la préservation de ces objets, il n'en demeure pas moins que le bon état de conservation du métal n'est sans doute pas sans rapport avec une bonne maîtrise de la cémentation par les forgerons de cette période. Dans certains cas, d'ailleurs, nous pouvons encore observer la trace des joints de grains de la microstructure du métal : il s'agit, en quelque sorte, d'une "structure métallique fantôme " ; cette continuité structurale entre le métal et l'oxyde est souvent considérée comme génératrice d'un effet protecteur de la gaine d'oxydes (Renoux, 2000 ; Renoux et al., 2001).

Le trait de catapulte est lui aussi fortement carburé. La bainite et la martensite, localisées sur les bords du carreau, témoignent d'un refroidissement rapide de la pièce après son forgeage ; la mise en cuvre délibérée d'une trempe à l'eau du trait n'est pas à exclure (Guillot, Fluzin, 1987 ; Guillot et al., 1987). Des traces de bainite ont pu être observées sur la pièce PM 502, mais nous pensons qu'elles résultent ici non d'un manque de maîtrise du forgeron, mais plutôt d'un incident lors de la fabrication, peut-être hâtive, de la pointe. Comme le suggèrent les écrits concernant la Guerre d'Afrique ${ }^{13}$, on peut penser que César ait pu ordonner la construction de forges, même rudimentaires, afin d'alimenter en armes le combat dont il voulait rapidement sortir victorieux. Cette thèse est accréditée par les textes antiques qui mentionnent que lors du siège d'Uxellodunum, l'armée romaine a pu construire une tour pour prendre d'assaut l'oppidum, ainsi que des mantelets et des fortifications tout autour de la place forte.

La technique délicate de la cémentation superficielle nécessite un savoir-faire adapté à l'obtention d'une bonne répartition du carbone dans des zones appropriées du métal. Elle est bien maîtrisée à l'époque romaine tout comme les opérations de refroidissement rapide (trempe) génératrices de phases durcissantes dans les aciers (martensite et bainite). En ce qui concerne cette étude sur les armes du Puy d'Issolud, le nombre encore trop restreint d'objets étudiés nous interdit toute conclusion définitive pour l'instant.

Sur quelques échantillons, nous avons rencontré des

\footnotetext{
${ }^{12}$ César, B.G., VIII, 42 (cf. chapitre 1).

${ }^{13}$ Ps- César, Bellum Africum, XXX, 3, Paris, Les Belles-Lettres, C.U.F., 1997, texte établi et traduit par A. Bouvet : «Il montait des forges, faisait fabriquer des flèches et des armes en grandes quantités, fondre des balles, préparer des piquets » (siège de Ruspina en 47 avant J.-C.).
} 
motifs en aiguilles de structure de Widmanstätten, fréquemment observées dans les fers anciens (fig. 3d). Cette morphologie est le produit d'un refroidissement assez rapide à l'air libre après un maintien à très haute température (entre $1000^{\circ} \mathrm{C}$ et $1100^{\circ} \mathrm{C}$ ). Les aiguilles de ferrite se développent selon des orientations bien définies, parallèlement à certains plans et directions cristallographiques des grains d'austénite (phase $\gamma$ ).

D'un point de vue exclusivement métallurgique, l'alternance de phases de chauffe et de déformation à chaud permet, par le jeu d'écrouissages et de recristallisations successifs, d'affiner la structure ferrito-perlitique, lui conservant ainsi un bon niveau de malléabilité. On peut imaginer que le traitement thermique ultime - celui conduisant à cette structure particulière dite de Widmanstätten- correspond pour un fer moyennement carburé $(0,5 \%$ C) à un bon compromis de dureté assez élevé (bon pouvoir de pénétration lors de l'impact), sans fragilisation excessive de l'objet (conservation d'une capacité de déformation plastique suffisante sans fissuration). Cette structure se rencontre dans les pointes de flèche PM 662 et sur le trait de catapulte mais très localement seulement.

\subsection{Microdureté}

Pour apprécier le niveau de résistance mécanique de ces armes, nous avons pratiqué des essais de microdureté Vickers (Hv) sous une charge de $50 \mathrm{~g}$. Les valeurs obtenues intègrent les relations entre la teneur en carbone, les phases présentes (ferrite, perlite, etc...) et la taille moyenne des cristaux. En règle générale, les valeurs de microdureté sont d'autant plus élevées que la teneur en carbone est forte et que, pour une teneur donnée, le refroidissement après forgeage est brutal et la taille des cristaux faible. L'hétérogénéité intrinsèque des structures observées provoque une dispersion sensible des valeurs de microdureté, nécessitant un nombre suffisant de mesures pour valider leur corrélation avec un état structural donné. Les résultats obtenus nous montrent que nous avons affaire à des aciers de bonne qualité, puisque les valeurs de $\mathrm{Hv}$ varient entre 118 et 255 . Les zones ferritiques sont, évidemment, moins dures que les domaines perlitiques pour lesquels nous obtenons dans certaines flèches des résultats dépassant $300 \mathrm{Hv}$, voire $335 \mathrm{Hv}$ pour la flèche PM 662. Les valeurs pour le trait de catapulte se situent entre $130 \mathrm{Hv}$ et $340 \mathrm{Hv}$ (valeurs pour des plages de martensite), confirmant ainsi le très haut niveau de résistance mécanique de cette pièce (fig. 4). Les valeurs obtenues pour la pointe de flècheBU10-484, retrouvée dans la zone incendiée, sont nettement plus faibles, puisqu'elles varient entre $81 \mathrm{Hv}$ et $143 \mathrm{Hv}$.

\subsection{La mise en forme des flèches}

Les examens métallographiques effectués permettent de proposer un mode de fabrication des pointes de flèche, notamment des flèches PM662 et PM594 à une barbelure. Il apparaît que l'extrémité de la pointe a été repliée et forgée à chaud pour faire partiellement corps avec l'axe principal de la flèche et créer ainsi la barbelure (fig. 5a et b, fig. 6). Les flèches BU10-484, BU10-438, BU10-706 et PM886, trop corrodées à leur extrémité, n'ont pu faire l'objet d'une étude aussi fine. Toutefois, dans tous les cas, l'examen des coupes transversales a permis de montrer que la douille était mise en forme par pliage conique de la partie inférieure de la flèche. Une autre méthode de fabrication des flèches à une barbelure a pu être observée, notamment sur la flèche inventoriée PM1054. En effet, la soudure que l'on peut observer est quasi parfaite, seul un très fin liseré blanc en matérialise la localisation. La microstructure observée de part et d'autre de la soudure est inchangée. Il s'agit donc de deux plaques en fer, assez carburées, qui ont été soudées pour former la barbelure (fig. $7 \mathrm{a}$ et $\mathrm{b}$ ). Le liseré clair correspond probablement à une décarburation très superficielle de l'acier avec formation d'un liseré de fer pur au niveau de la surface de soudage.

Il est connu que le soudage de deux plaques de fer nécessite une grande habileté de la part du forgeron. Plusieurs facteurs entrent en jeu : la température de fusion,

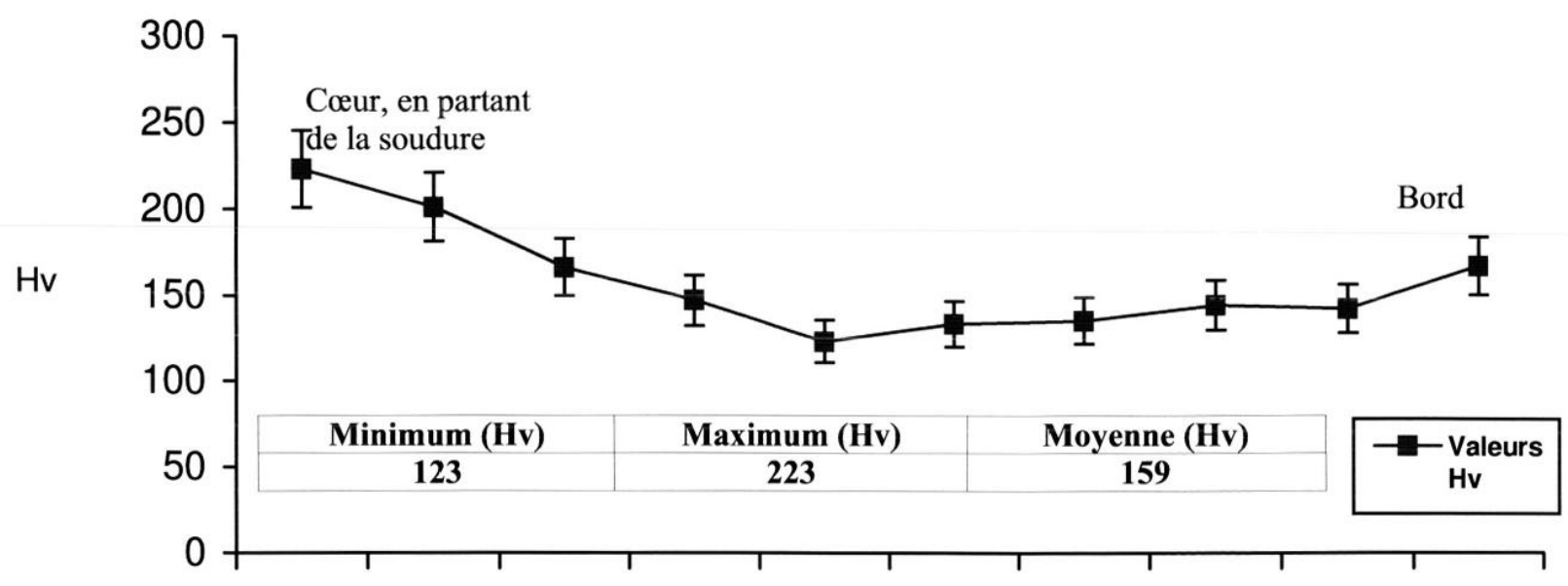

Distance entre chaque mesure $(100 \mu \mathrm{m} /$ division $)$

Figure 4 : Valeurs de microdureté Vickers Hv sous une charge de $50 \mathrm{~g}$, sur la pointe de flèche PM 594. Figure 4: Values of Vickers microhardness (Hv), with a charge of $50 \mathrm{~g}$., observed with the arrow head PM 594. 


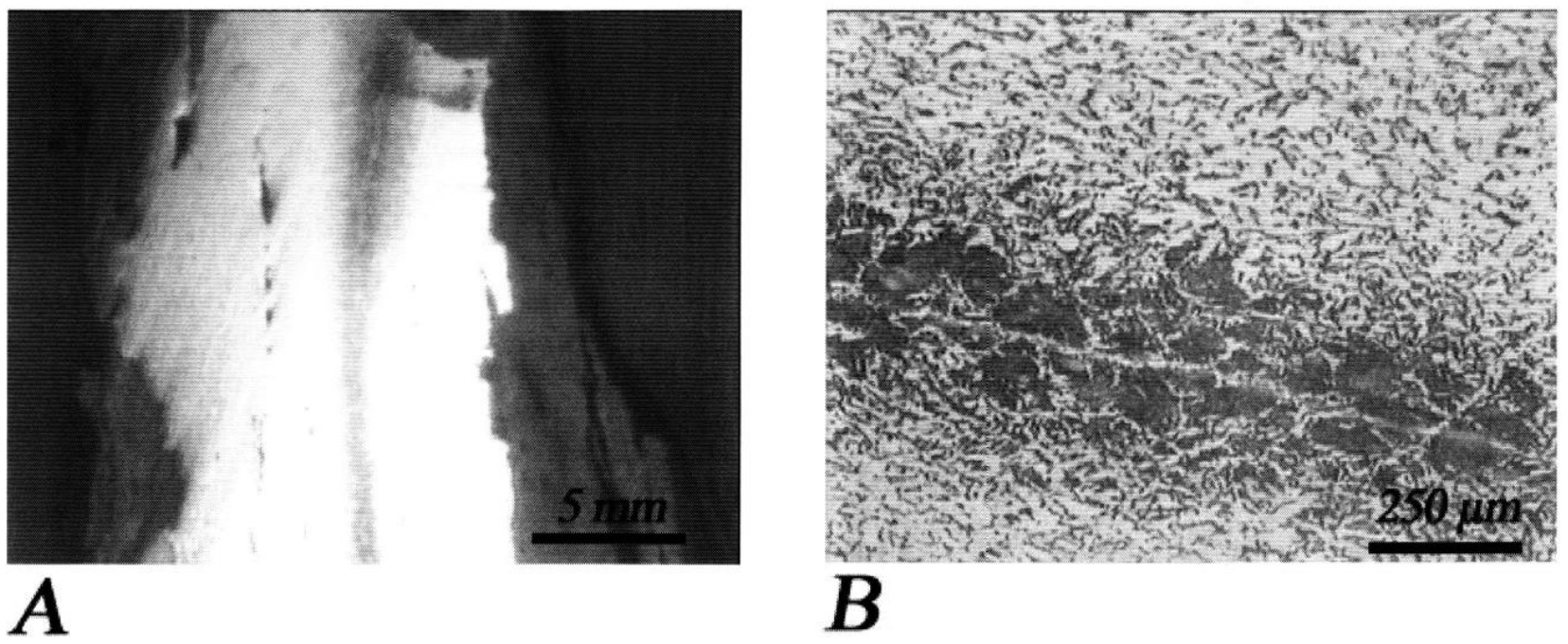

Figure 5 : A : Macrographie optique. Pointe de flèche PM594. Soudure de la barbelure.

B : Micrographie optique. Pointe de flèche à une barbelure PM 594. Structure ferrito-perlitique avec bande blanche au nivcau du soudage partiel de la barbelure lors du forgeage. Structure ferritique vers l'cxtéricur de la zonc.

Figure 5: A: Optical macrograph. Arrow head PM 594. Welding zone of the barbelure.

B: Optical micrograph. Arrow head with a barbelure PM 594. Ferrito-perlitic structure with a white border at the partial welding junction of the barbelure, developped during the forging operation. Ferritic structure at the outer part of this zone.
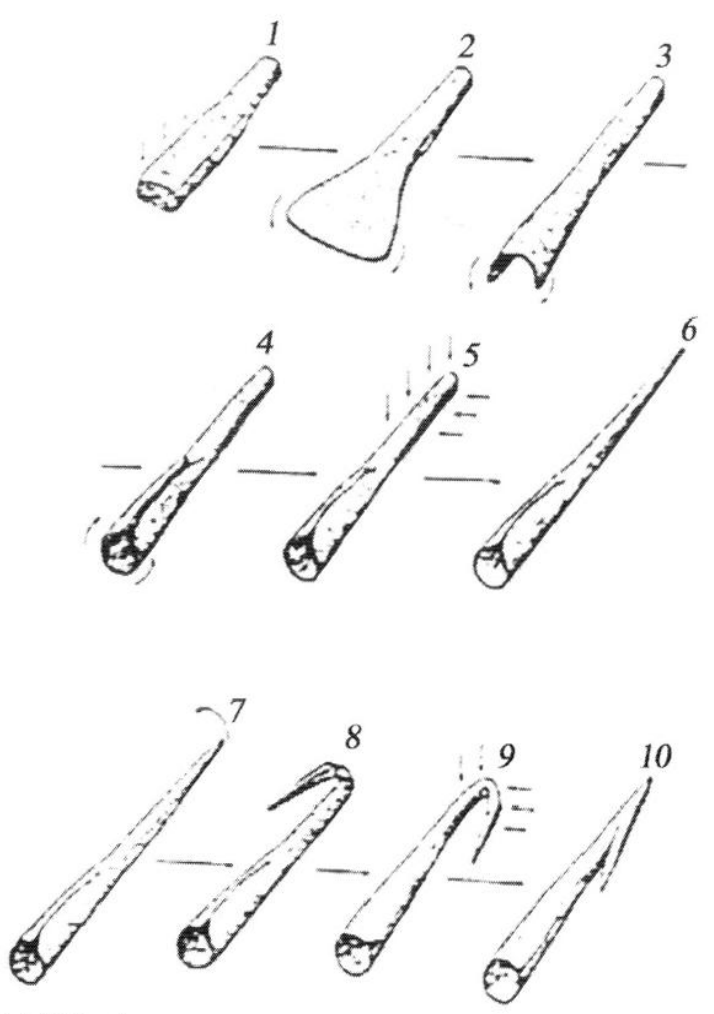

D'après J.-P. Girault

Figure 6: Principales étapes de réalisation des pointes de flèche droite $\left(\mathrm{n}^{\circ} 1\right.$ à 6$)$ et de flèche à une barbelure $\left(\mathrm{n}^{\circ} 7\right.$ à 10$)$.

Figure 6: Main steps of process involved for doing right arrow head $\left(n^{\circ}\right.$ 1 to 6) andarrow head with a barbelure ( $n^{\circ} 7$ to 10$)$.

la teneur en carbone ainsi que l'oxydation à haute température (Guillot, Fluzin, 1987 ; Guillot et al., 1987). La liaison de soudage - très probablement réalisée ici par diffusion à l'état solide - entre deux surfaces très propres amenées au contact l'une de l'autre nécessite avec le fer pur, né d'une décarburation très superficielle, des températures très élevées (de l'ordre de $1400^{\circ} \mathrm{C}$ ) (Fluzin et al., 2000) que le forgeage permet sans doute d'abaisser autour de $1300^{\circ} \mathrm{C}$; cette dernière valeur assortie d'une ambiance décarburante est compatible avec l'aire de travail du forgeron. On notera que le palier de soudabilité (domaine de température permettant le soudage) dépend de la nature du métal à assembler. Ce palier est plus haut pour le fer pur que pour un acier. Il est donc plus difficile de réaliser une soudure acier/fer que acier/acier, par exemple, car il faut que les deux métaux soient amenés en même temps à leur palier de soudabilité. Il semblerait que ce ne soit pas le cas ici.

Les microstructures rencontrées montrent, aussi bien pour les flèches ayant l'extrémité recourbée que pour celle dont la barbelure a été formée par soudage de deux morceaux en fer, que les pièces métalliques utilisées étaient carburées, subissant seulement une très faible décarburation au moment du soudage-forgeage. Le forgeron doit également s'attacher à éviter la formation d'oxydes entre les deux pièces qui s'opposerait à la liaison de ces dernières. Pour cela, il utilise des décapants (argile, sable, poudre de scories) qui forment un film de silicate à haute température protégeant ainsi la surface des deux pièces métalliques (Fluzin et al., 2000). Les analyses EDXS couplées aux images réalisées au MEB montrent très nettement que des oxydes résiduels, formant des alignements d'inclusions, se trouvent dans le plan de soudure (fig. 8). En général, le forgeron ne peut éviter totalement la formation de ces oxydes.

\subsection{Les inclusions : les différents types de scories}

Les résultats des analyses des scories incluses dans le métal constituent de précieux indices pour tenter de retracer l'histoire du métal, tant au stade de son élaboration qu'à celui du forgeage. Deux catégories de scories ont été différenciées. Les unes présentent des morphologies internes dendritiques tandis que les secondes, apparemment monophasées, se révèlent vitreuses (Dillmann, 1998). 

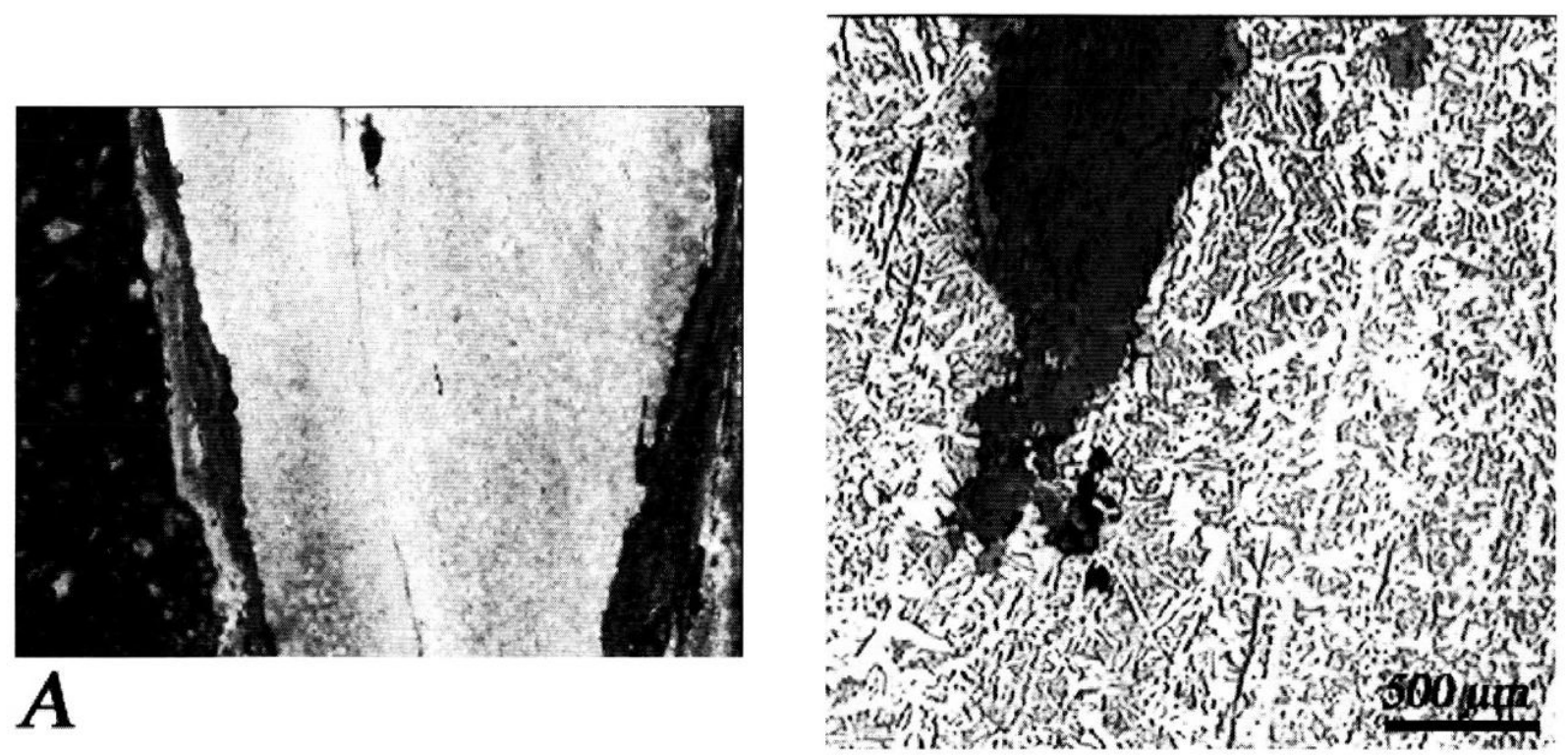

B

Figure 7 : A : Macrographic optique. Soudure de la barbelure de la pointe de flèche PM 1054.

$\mathrm{B}$ : Micrographic optique. La bande blanche an centre représente la soudure. Structure ferrito-perlitique de part et d'autre de la soudure. Figure 7: A: Optical macrograph. Welding of the barbelure of the arrow head PM 1054.

B: Optical micrograph. The central clear band represents welding. Ferrito-perlitic structure on the sides of the welding zone.

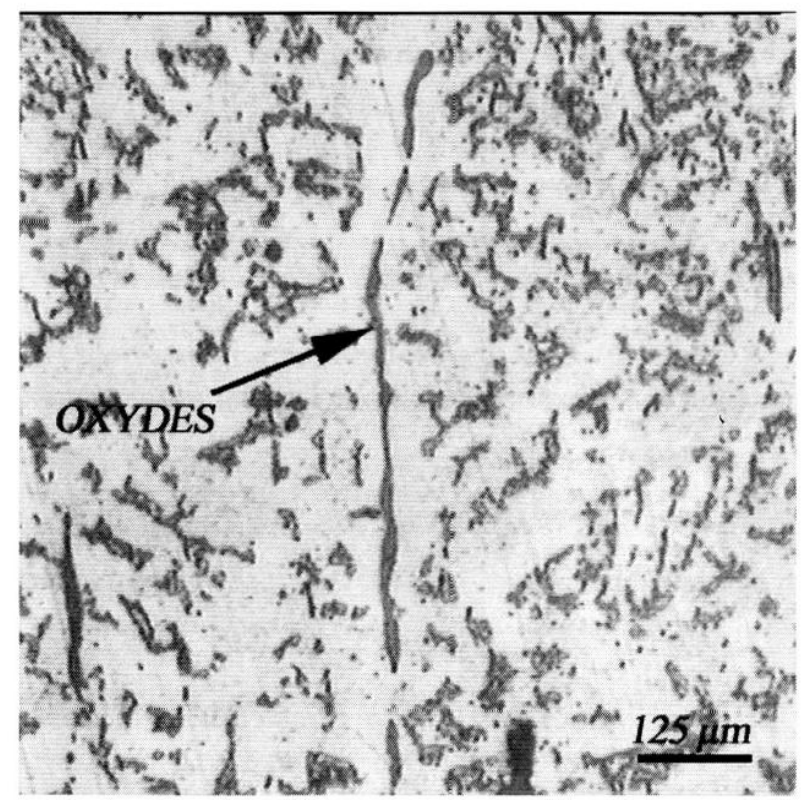

Figure 8 : Micrographic optique. Pointe de flèche PM 1054. Présence d'oxydes résiducls formant des alignements dans le plan de la soudurc.

Figure 8: Optical micrograph. Arrow head PM 1054. Residual oxide particles delineate stringers in the welding plane.

Les scories en dendrites, riches en fer, sont issues des opérations de réduction directe du minerai de fer en bas fourneau. La structure dendritique, associée aux cristaux de wüstite $(\mathrm{FeO})$, résulte de la rétention dans la scorie liquide d'une partie du fer du minerai. Lors de la solidification de celle-ci, la croissance dendritique de la wüs- tite se produit naturellement au sein d'une phase silicatée (fayalite $\mathrm{Fe}_{2} \mathrm{SiO}_{4}$ ) encore liquide. Au moment du cinglage, des résidus de charbon de bois et de scories peuvent subsister dans la loupe de métal. Nous les retrouvons donc sous forme d'inclusions dans la pièce forgée.

Les scories vitreuses, à teneur en fer beaucoup plus faible (teneur égale ou inférieure à $10 \%$ ), renferment, comme les précédentes, divers éléments - aluminium $(\mathrm{Al})$, silicium $(\mathrm{Si})$, potassium $(\mathrm{K})$, calcium $(\mathrm{Ca})$, manganèse $(\mathrm{Mn})$ - identifiés par analyses EDXS au MEB (Domergue, Tollon, 1973). L'origine de ces scories est majoritairement associée aux adjuvants (chaux, sable, argile) introduits lors du forgeage pour affiner la loupe, en éliminant, si possible, la totalité des scories résiduelles ; mais cela est rarement le cas et nous retrouvons donc dans le métal des inclusions silicatées, témoins de l'opération de mise en forme (fig. 9a et b). La présence de ces éléments peut, en partie, résulter aussi de la réaction du revêtement réfractaire du bas fourneau avec le minerai (Dillmann, 1998 ; Domergue, 1995 ; Mangin et al., 2000a et 2000b).

Il est donc très utile d'examiner de façon approfondie ces deux types de scories qui peuvent, par la nature des impuretés et leurs teneurs, fournir de précieuses informations d'une part sur les types de minerais utilisés, leur origine (sites d'exploitation) et les caractéristiques de la méthode de production et d'autre part, sur les opérations de forgeage (Piaskowski, 1984).

Dans cette démarche, l'étude des fers de traits de catapulte, arme spécifiquement romaine ${ }^{14}$ (Sim, 1992, 1995),

\footnotetext{
${ }^{14}$ Pour les flèches, il scrait impropre de les qualifier de « romaines » ou de « gauloises ». Etant donné les conditions de la bataille, ces flèches proviennent pour l'essentiel du camp romain. Mais bon nombre d'entre clles ont pu être fabriquées sur place, ou à proximité, par des forgerons gaulois intégrés ou associćs à l'arméc de César.
} 

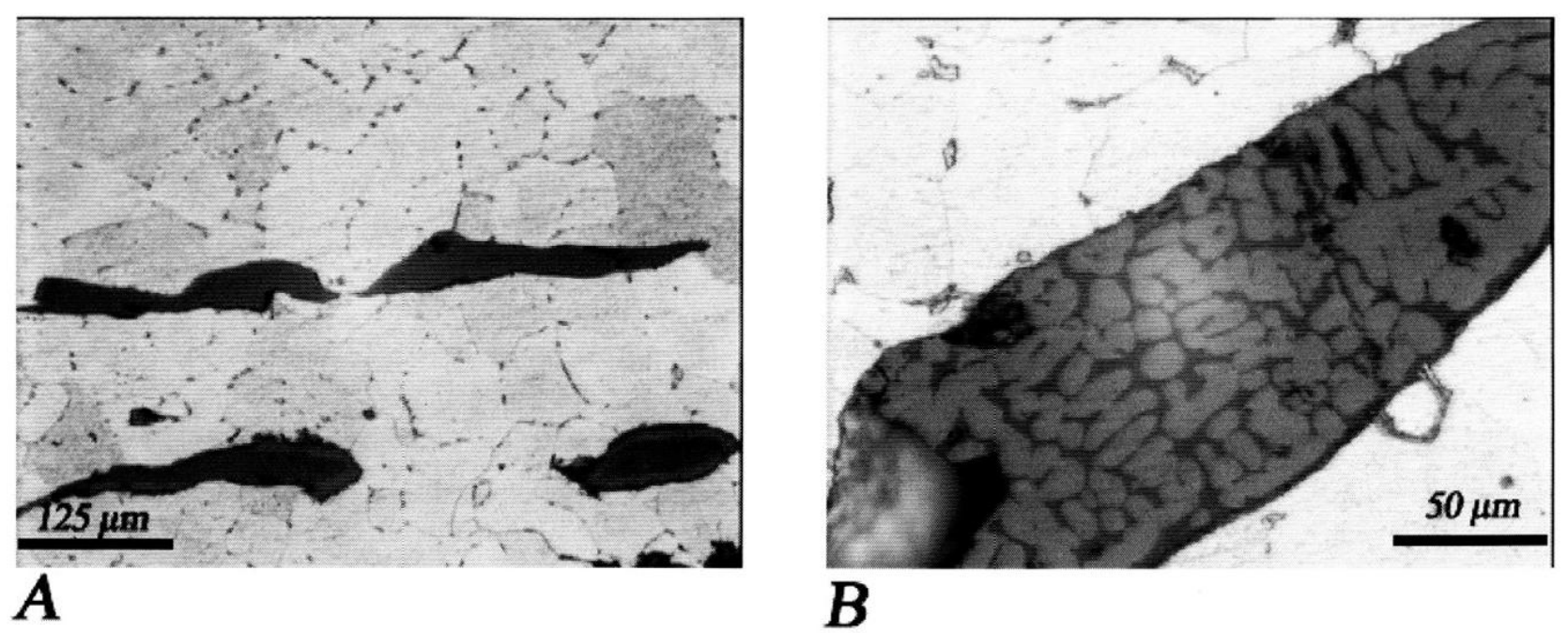

Figure 9 : Micrographies optiques. Exemples d'inclusions. Ces différents types de scories se retrouvent au cœur même des flèches.

A : Scories monophasées présentes dans les flèches PM 594 et PM1054.

$\mathrm{B}$ : Scoric à structure dendritique de wüstite $\mathrm{FeO}$, présentes dans la fièche PM 594

Figure 9: Optical micrographs. Examples of slag inclusions; these different types of slags are found in the core of arrows.

A: Monophased slags, found in the arrow heads PM 594 and PM 1054.

$B$ : Slags with a dendritic structure of wustite $\mathrm{FeO}$, observed in the arrow head PM 594.

constitue une référence particulièrement utile pour identifier l'origine des pointes de flèche; l'analyse de leurs scories, inventoriant avec précision leurs éléments constitutifs, peut permettre d'intéressantes comparaisons avec les compositions de celles relatives aux pointes de flèche. La confrontation des résultats doit permettre de mieux expliciter les origines de ces armes et, pour le moins, d'affiner les connaissances techniques sur leur mise en forme. Il sera également souhaitable de procéder dans les mêmes conditions expérimentales à des études semblables sur des objets de même type provenant d'autres sites archéologiques de même époque ${ }^{15}$.

Toutes ces études métallographiques ont été complétées par des analyses à la microsonde ionique sur des échantillons prélevés, notamment, sur la pointe de flèche BU10-484. Nous avons pu mettre en évidence notamment la répartition du carbone dans la matrice du fer composant cette flèche. Ce dernier, se situant aux joints de grains, matérialise ainsi les précipités de cémentite précédemment cités.

\section{Conclusion}

Les examens et les analyses classiques d'objets archéologiques métalliques, fondées essentiellement sur leurs caractères extérieurs (typologie), peuvent être considérablement enrichis par un examen de leur morphologie interne qui permet, en dépassant les simples fonctions d'utilisation, d'accéder aux techniques de forgeage et aux modes de fabrication de ces types d'objets dans l'Antiquité.

Si les textes constituent un témoignage direct de la vie des hommes dans l'Antiquité, le mobilier que nous étudions forme, quant à lui, un support complémentaire de notre connaissance du passé. C'est ainsi que, sans négliger l'apport des sources écrites et archéologiques, qui fournissent ici des renseignements irremplaçables sur la chronologie et sur les circonstances, la métallographie permet d'élargir le champ des investigations analytiques et microstructurales ; c'est l'objectif de nos recherches sur les armes du Puy d'Issolud. Si nous savons par quelques textes que les forgerons étaient intimement liés aux armées romaines, comme le précise notamment Végèce ${ }^{16}$, nous souffrons par contre d'un manque d'écrits sur les techniques de forgeage qu'ils utilisaient. Les analyses métallurgiques ainsi que l'archéologie expérimentale ${ }^{17}$ (Leblanc, 2002) pourront à la fois nous apporter des renseignements sur ces techniques mais aussi sur l'évolution de l'armement.

\section{Bibliographie}

DILLMANN, Ph., 1998 - Diffraction X, Microdiffraction X et Microfluorescence $X$ sous Rayonnement Synchrotron et analyses comparées pour la caractérisation des inclusions. Application à l'étude de l'évolution historique des procédés d'élaboration des objets ferreux (procédés direct et indirect), Thèse de doctorat de l'Université de Technologie de Compiègne, 2 vol.

\footnotetext{
${ }^{15}$ Dans le même esprit nous avons entrepris une étude comparative sur des clous de souliers et de charpentes, provenant du Puy d'Issolud, d'Alésia ainsi que des Martys. Tous ces objets datent du Ier s. av. J.-C.

${ }^{16}$ Végèce, II, 11 « La légion a à sa suite des menuisiers, des maçons, des charpenticrs, des forgerons [...] lls avaient des ateliers pour les boucliers, les cuirasses et les arcs, dans lesquels ils fabriquaient aussi des flèches, des javclots, des casques et toutes sortes d'armes. »

${ }^{17}$ Dans le cadre du Colloque Intcrnational « Orfèvres et forgerons. L'approche expérimentale en Archéologie minière et métallurgique », organisć en Octobre 2001 à l'Université de Toulousc-Lc Mirail, Mr Hector Cole, forgeron et expérimentateur anglais, a pu montrer comment certains types de flèches d'époque romainc, notamment les types $\mathrm{A}$ et $\mathrm{B}$, étaient fabriquées.
} 
DOMERGUE, Cl., TOLLON, F., 1973 - « Mincrais et scorics de la fonderie gallo-romaine du domaine des forges. Les Martys (Aude) », $98^{e}$ Congrès national des Sociétés savantes, Saint-Etienne, section archéologic, 101-114.

DOMERGUE, Cl., 1995 - « Les fourncaux de réduction du fer du I ${ }^{\text {r }}$ sic̀cle av. J.-C. découverts aux Martys (Aude) », in Objets en fer ...et savoir-faire, Rencontres Archéologiques de Guiry-en-Vexin, Actes des journées Autour du fer, avril 1992-1993, Muséc Archéologiquc Départemental du Val-d'Oise, 10-12.

DUVAL, A., 1970 - «Les pointes de flèche d'Alésia au Musće des Antiquités Nationales », Antiquités Nationales, St-Gcrmain-enLaye, 2, 35-51.

FLUZIN, Ph., URAN, L., CODDET, C., BERANGER, G., 1983 « Structures métallurgiques des armes de Gournay-sur-Aronde : lecturc et interprétation ", Journées de paléométallurgie, Universitć dc Technologic de Compiègne, 85-101.

FluZIN, Ph., PloQuin, A., SERNEels, V., 2000 - «Archćométric des déchets de production sidérurgique : moyens et méthodes d'identification des différents éléments de la chaîne opératoire directe ", Gallia, 57, 101-121.

GUILLOT, I., FLUZIN, Ph., 1987 - «Interprétation structuralc dc l'ćlaboration ct de l'utilisation d'outils minicrs ", Bulletin de la Société Préhistorique Française, 84, 8, 248-256.

GUILlOT, I., FLUZIN, Ph., CLAVEL, M., BERANGER, G., 1987 "Structure d'outils minicrs du XV' et XVIII' siècle. Interprétation thermomécanique ", Matériaux et Techniques, 10-11, 411-419.

LEBLANC, J.-Cl., 2002 - Sur l'identification de la chaîne opératoire de forgeage du fer antique, associée à l'archéométrie des battitures, thc̀sc de doctorat de l'Universitć de Toulouse III - Paul Sabatier, 2 vol.

LEJARS, T., 1989 - « Les armes des sanctuaircs poitevins d'ćpoque préromaine de Fayc-l'Abbesse (Dcux-Sèvres) et de Nalliers (Vendéc) », Gallia, $n^{\circ} 46,1-41$.

MANGIN, M., CORTADON, J.-L., FLUZIN, Ph., De LACLOS, E., 2000a - Village, forges et parcellaire aux sources de la Seine. L'agglomération antique de Blessey-Salmaise (Côte d'Or), Pressc Universitairc Franc-Comtoisc, Annales littćraircs de l'Université, Séric « Environnement, Socićtćs, Archéologic » n².
MANGIN, M., FLUZIN, Ph., CORTADON, J.-L., FONTAINE, M.J., 2000b - Forgerons et paysans des campagnes d'Alésia (HautAuxois, Côte-d'Or). La terre, le fer, la route en pays mandubien ( ${ }^{\text {er }}$ s. av.-VIII ime s. ap. J.-C.), C.R.A. 22 Monographies, C.N.R.S Editions, Paris, $508 \mathrm{p}$

MOHEN, J.-P., 1973 - «Qu'attendre de la radiographic des objets protohistoriques en Bronze ", Bulletin de la Société Préhistorique Française, 70, 205-210.

PAJOT, B., 1978 - « Radiographics de quelques ćpées à antennes dc la rćgion Midi-Pyrćnćcs ", Bulletin de la Société préhistorique française, Tome 75, n 11-12, C.N.R.S., 610-623.

PIASKOWSKI, J., 1984 - «Proposals for a Standardization of the Criteria for Determining Technological Processes in Early and Steel Metallurgy ", in The Crafts of the Blacksmith, Ed. B.G. Scott et H. Clccrc, Symposium 1984, Comité U.I.S.P.P., Belfast, 157-168.

RENOUX, G., 2000 - Recherches sur les armes en fer découvertes au Puy d'Issolud (Lot) : contexte archéologique et étude paléométallurgique, Mémoirc de D.E.A. Sciences de l'Antiquité, Université de Toulouse-Lc Mirail, $130 \mathrm{p}$.

RENOUX, G., PAILlER, J.-M., DABOSI, F., 2001 - «Premièrc étude palćométallurgique des armes en fer du Puy d'Issolud », Revue de Métallungie-CIT/Science et Génie des Matériaux, tome 98, ${ }^{\circ} 12$ (décembre), 1147-1158.

SERDON, V., 2002 - « Etude palćomćtallurgique de fers de traits du Moyen-Age, contribution à l'histoire des techniques ", Revue d'Archéométrie, 26, 209-218.

SERDON, V., 2003 - L 'armement de trait au Moyen Age. Etude archéologique, $X I^{\text {ème }}-X V^{\text {ème }}$ siècle, Thèse de doctorat, Université Lumière Lyon II.

SIM, D., 1992 - « The manufacturc of disposable wcapons for the Roman army ", Journal of Roman Military Equipment Studies, vol. 3, 105-119.

SIM, D., 1995 - « Wcapons and mass production », Journal of Roman Military Equipment Studies, vol. 6, 1-3.

URAN, L., 1986 - «Observation métallographique sur les épées en fer », Aquitania, Supplément 1, 299-309. 